\title{
Convergence Analysis for Linear Turbo Equalization
}

\author{
Seok-Jun Lee and Andrew C. Singer \\ Coordinated Science Laboratory, ECE Dept. \\ University of Illinois at Urbana-Champaign \\ 1308 West Main Street, Urbana, IL 61801 \\ Email: [slee6,acsinger]@uiuc.edu
}

\begin{abstract}
In this paper, we propose a method for analysis of linear turbo equalization that makes use of extrinsic information transfer (EXIT) charts. Given channel knowledge and a timeinvariant set of linear equalizer coefficients, evolution of soft information in the soft-input soft-output (SISO) equalizer can be estimated via analytically measuring the mutual information between the transmitted symbols and their estimated a priori values at the two end points of an EXIT chart. Using this estimated equalizer EXIT chart and an empirically generated decoder EXIT chart, convergence analysis can be undertaken. In comparison with existing EXIT chart based methods, the proposed approach can significantly reduce the reliance on extensive computer simulation.
\end{abstract}

\section{INTRODUCTION}

Since the discovery of turbo codes [1], the methods developed for iterative decoding of such codes have influenced a wide variety of applications [2], [3]. Of these applications, linear turbo equalization [4]-[6] has gained significant interest, since it can mitigate inter-symbol interference (ISI) effectively with reasonable complexity. -

Recently, a number of results on the analysis of turbo decoding and turbo equalization [5], [7]-[11] have been presented in the literature. Many such analysis methods trace the convergence of iterative decoding algorithms by monitoring a single parameter across multiple iterations. This parameter is assumed to capture the salient characteristics of the behavior of the soft-input soft-output (SISO) blocks which are employed in turbo receivers. Such parameters are mutual information between the transmitted symbol and its soft information computed in SISO decoders [5], [7], [8] (which is of particular interest to this paper), estimated noise variance [9], the probability density function of the log-likelihood ratio (LLR) [10], [11], and a number of other related metrics.

However, a drawback of existing analysis methods based on single parameters is the reliance on extensive computer simulations in order to obtain the parameter evolution. In this paper, the evolution of one such parameter (the mutual information between the transmitted symbol and its soft information computed in the linear SISO equalizer) is estimated without running extensive simulations. Given channel knowledge and a time-invariant set of equalizer coefficients, the mutual information at the two end points of an extrinsic information transfer (EXIT) chart can be analytically computed. By approximating the EXIT chart of the SISO equalizer to be linear [5], [7], the two end-point values can be used to predict the soft information evolution on the EXIT chart. Using such

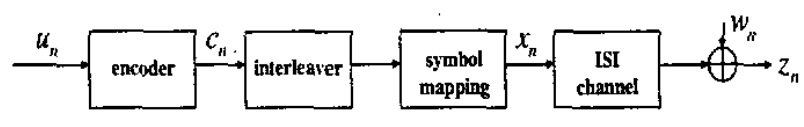

Fig. 1. A typical transmit model.

a method, convergence analysis can be undertaken and the potential gains from turbo equalization rather than a traditional non-iterative receiver can also be determined.

The rest of this paper is organized as follows. After a review of linear turbo equalization and EXIT charts in the next section, the mutual information at the two end points of the linear SISO equalizer EXIT chart is derived and our analysis method is explained in Section III. Simulation results and some discussions are provided in Section IV, and Section $\mathrm{V}$ concludes this paper.

\section{LINEAR TURBo EQUALIZER AND EXIT CHARTS}

This section briefly describes linear turbo equalization [4], [5], and an analysis tool, called an EXIT chart, for convergence analysis of turbo equalization [5], [8].

\section{A. Linear Turbo Equalization}

For simplicity, we assume binary phase shift keying (BPSK) modulation, but extension to higher-order modulation is straightforward and is described in greater detail in [6].

The system to be investigated has a transmitter as depicted in Fig. 1 with block-based transmission. The binary data $u_{n}$ is encoded yielding the coded sequence $c_{n}$, which the interleaver permutes. Then BPSK modulated symbols $x_{n} \in\{-1,+1\}$ are transmitted over an ISI channel with additive white Gaussian noise (AWGN). The channel output $z_{n}$ is given by

$$
z_{n}=\sum_{k=-l_{1}}^{l_{2}} h_{k} x_{n-k}+w_{n}
$$

for a channel response $h_{k}$ with length of $l_{1}+l_{2}+1$ and noise sequence $w_{n}$.

Figure 2 depicts a typical architecture of the linear turboequalizer [4], [5], where the superscript $E$ and $D$ denote equalization and decoding, respectively, and the subscript $o$ and $i$ mean the output and input, respectively. This linear SISO equalizer consists of two operations: symbol estimation and soft-information mapping of estimated symbols. The first operation, estimation, is implemented via a linear filter, and the overall architecture is similar to that of the well-known 


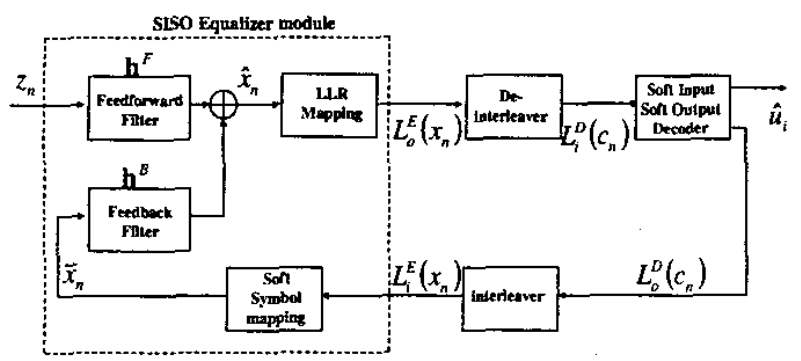

Fig. 2. A linear turbo-equalizer block diagram, in which soft information is exchanged between the equalizer and decoder.

decision-feedback equalizer (DFE). However, instead of hard quantized decisions, soft symbols $\bar{x}_{n}$ are passed to a feedback filter from the previous iteration of the SISO decoder. The LLR mapping converts each estimated symbol $\hat{x}_{n}$ to a LLR $L_{o}^{E}\left(x_{n}\right)$, which is calculated as

$$
L_{o}^{E}\left(x_{n}\right)=\ln \frac{\operatorname{Pr}\left(\hat{x}_{n} \mid x_{n}=+1\right)}{\operatorname{Pr}\left(\hat{x}_{n} \mid x_{n}=-1\right)}
$$

for BPSK signals.

The updated $L_{o}^{E}$ are fed to the SISO decoder after deinterleaving, and the decoder improves the soft information on coded bits, $c_{n}$, and produces $L_{o}^{D}$, the LLR of each coded bit. In turn, $L_{o}^{D}(\cdot)$ is passed to the interleaver and used as input to the soft symbol mapping block, which converts $L_{i}^{E}\left(x_{n}\right)$ to the soft symbol $\bar{x}_{n}$, computed as

$$
\begin{gathered}
\bar{x}_{n}=E\left\{x_{n}\right\}=\operatorname{Pr}\left\{x_{n}=1\right\}-1+\operatorname{Pr}\left\{x_{n}=-1\right\} \cdot(-1) \\
\bar{x}_{n}=\frac{\exp \left(L_{i}^{E}\left(x_{n}\right)\right)-1}{1+\exp \left(L_{i}^{E}\left(x_{n}\right)\right)}
\end{gathered}
$$

where $E\{\cdot\}$ denotes a statistical expectation. This soft symbol is then fed back to the equalizer block for the next iteration. The details of such a SISO decoder algorithm are described in [12].

\section{B. The EXIT chart}

The EXIT chart is a tool used to trace the convergence of iterative algorithms by observing the behavior over iterations of a single parameter. In Ten Brink's approach [8], the parameter is the mutual information $I_{i}$ and $I_{o} \in[0,1\}$, between $a$

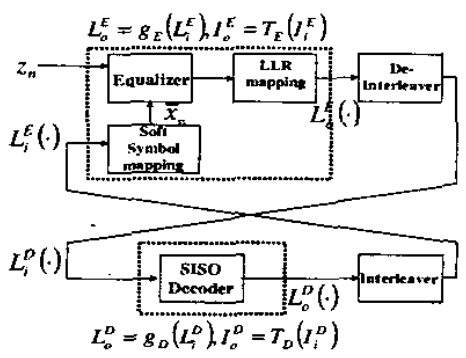

Fig. 3. Modeling $L_{i}, L_{o}$-value update as transfer function.

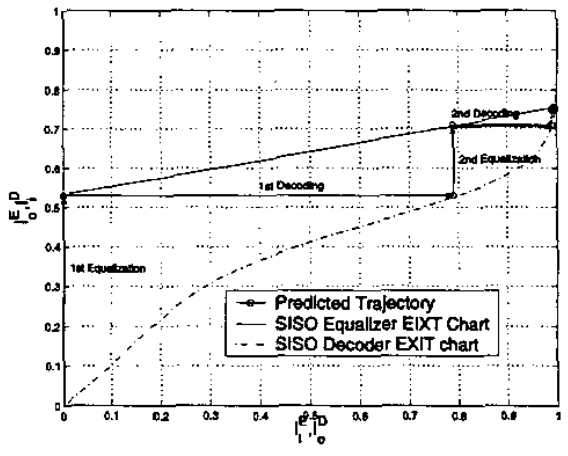

Fig. 4. An example of EXTT chart, in which the EXIT charts of SISO equalizer and decoder are obtained experimentally.

priori values $\left(L_{i}\right.$ or $L_{o}$ ) and $x_{n}$, which is shown to describe the behavior of SISO algorithms accurately [5], [7].

In [5], [8], $L_{i}(\cdot)$ is assumed to have a Gaussian distribution of the form $\mathcal{N}\left(\frac{x_{n} \sigma_{L_{i}}^{2}}{2}, \sigma_{L_{i}}^{2}\right)$ and the histogram of the outputs $L_{o}$ is used to estimate the probability density function (pdf) of $L_{o}$. Then, $I_{i}$ and $I_{o}$ are computed numerically using

$$
I=\frac{1}{2} \sum_{x \in\{ \pm 1\}} \int f_{L}(l \mid x) \log _{2} \frac{2 f_{L}(l \mid x)}{f_{L}(l \mid+1)+f_{L}(l \mid-1)} d l,
$$

where, $f_{L}\left(l \mid x_{n}\right) \triangleq f_{L}\left(l \mid X=x_{n}\right)$. Note that $I_{i}=0$ and $I_{i}=$ 1 , mutual information between input a priori information and $x_{n}$, imply no and perfect a priori information, respectively. $I_{\mathrm{o}}=0$ and $I_{\mathrm{o}}=1$, mutual information between output $a$ priori information and $x_{n}$, indicate the least and the most reliable soft output information.

In an EXIT chart, the mutual information evolution can be visualized as a transfer function, $I_{o}^{E}=T_{E}\left(I_{i}^{E}=I_{o}^{D}\right)$ or $I_{o}^{D}=T_{D}\left(I_{i}^{D}=I_{o}^{E}\right)$ as shown in Fig. 3 [5]. Then, the iteration process can be modeled as a trace (see Fig. 4) between the EXIT charts of the equalizer and decoder by setting $I_{o}^{E} \longrightarrow$ $I_{i}^{D}$ and $I_{o}^{D} \longrightarrow I_{i}^{E}$.

Figure 4 shows an example EXIT chart analysis of turbo equalization. If the equalizer generates soft information $\left(I_{o}^{E}=\right.$ 0.53 ) in the first iteration, then the decoder produces an improved soft output $\left(I_{o}^{D}=0.78\right)$. This computation can be traced by the arrow-line in Fig. 4 and after 3 iterations, the $I_{o}^{D}=1$ condition, corresponding algorithmic convergence, is nearly achieved. Note that, in order for this graphical depiction of convergence to succeed in achieving the $I_{o}^{D}=1$ point, a "tunnel" between the two EXIT charts of the equalizer and decoder must appear.

\section{ANALYSIS}

In this section, the mutual information between the transmitted symbol sequence and the LLR output sequence of the linear SISO equalizer is derived. The proposed analysis method is then explained. 


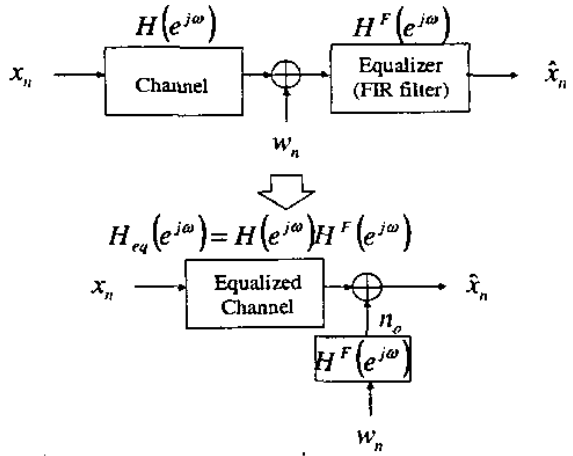

Fig. 5. Conventional communication system with a linear equalizer, where $H\left(e^{j \omega}\right), H^{F}\left(e^{j \omega}\right)$, and $H_{e q}(z)$ are frequency domain responses of a channel, a linear equalizer, and an overall response after equalization, respectively.

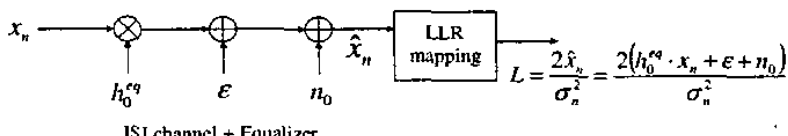

Fig. 6. Equalized channel model for measuring the SISO equalizer EXIT charts, where $h_{0}^{\epsilon q}$ is the main cursor in the equalized channel response and $\epsilon$ is the ISI effect caused by neighboring symbols of the current symbol $x_{n}$.

\section{A. Mutual information derivation}

Figure 5 depicts the model considered in this analysis for a given channel impulse response and equalization algorithm (a time-invariant set of filter coefficients). The responses of the channel and the equalizer are combined such that the output power spectral density $S_{\hat{x} \hat{x}}\left(e^{j \omega}\right)$ can be written as

$$
\begin{aligned}
S_{\hat{x} \hat{x}}\left(e^{j \omega}\right) & =\left|\mathbf{H}\left(e^{j \omega}\right)\right|^{2}\left|\mathbf{H}^{F}\left(e^{j \omega}\right)\right|^{2} S_{x x}\left(e^{j \omega}\right) \\
& +\left|\mathbf{H}^{F}\left(e^{j \omega}\right)\right|^{2} S_{w \psi}\left(e^{j \omega}\right),
\end{aligned}
$$

where $S_{x x}\left(e^{j \omega}\right)$ is the input power spectral density and $S_{w w}\left(e^{j \omega}\right)$ is the power spectral density of the noise process $w_{n}$, which is assumed white and uncorrelated with $x_{n}$, i.e. $S_{w i v}\left(e^{j \omega}\right)=1$. The estimated symbol $\hat{x}_{n}$ is then given by

$$
\hat{x}_{n}=\left(\mathbf{h} * \mathbf{h}^{F}\right)^{T} \cdot \mathbf{x}+n_{o}=\left(\mathbf{h}^{e q}\right)^{T} \cdot \mathbf{x}+n_{o},
$$

where $*$ denotes convolution, $\mathbf{h}=$ $\left[h_{-l_{1}}, \cdots, h_{-1}, h_{0}, h_{+1}, \cdots, h_{+l_{2}}\right]^{T}, \quad \mathbf{h}^{e q}$ denotes the equalized channel response, the filtered noise sequence $n_{o}$ has variance $\sigma_{n_{o}}^{2}=\sigma_{w}^{2} \sum_{k}\left|h_{k}^{F}\right|^{2}, \mathbf{x}=$ $\left[x_{n+M_{1}}, \cdots, x_{n+1}, x_{n}, x_{n-1}, \cdots, x_{n-M_{2}}\right]^{T}, M_{1}=L_{1}+l_{1}$, and $M_{2}=L_{2}+l_{2}$. By defining

$$
\mathbf{h}^{e q 0} \triangleq\left[h_{-M_{1}}^{e q}, \cdots, h_{-1}^{e q}, 0, h_{+1}^{e q}, \cdots, h_{M_{2}}^{e q}\right]^{T},
$$

the estimated symbol can be expressed as

$$
\hat{x}_{n}=h_{0}^{e q} \cdot x_{n}+\left(\mathbf{h}^{e q 0}\right)^{T} \cdot \mathbf{x}+n_{o}=h_{0}^{e q} \cdot x_{n}+\epsilon+n_{o},
$$

where $\epsilon$ is the ISI caused by neighboring symbols and is deterministic given a set of transmitted symbols neighboring $x_{n}$. Thus, the equalized channel can be conditionally modeled with one constant multiplication and one constant addition as shown in Fig. 6. The LLR mapping is assumed to have the functionality, $L\left(\hat{x}_{n}\right) \triangleq \frac{2 \hat{x}_{n}}{\sigma^{2}}$, as in a memoryless channel with AWGN or in the case of perfect equalization [8]. Using this mapping, the LLR of the linear SISO equalizer becomes

$$
L_{o}^{E}=\ln \frac{p\left(\hat{x}_{n} \mid x_{n}=+1\right)}{p\left(\hat{x}_{n} \mid x_{n}=-1\right)}=\frac{2 \hat{x}_{n}}{\sigma_{n_{o}}^{2}}=\frac{2\left(h_{0}^{e q} x_{n}+\epsilon+n_{o}\right)}{\sigma_{n_{o}}^{2}},
$$

where the LLR values are identical to the AWGN channel case if $h_{0}^{e q}=1, \epsilon=0$, and $\left|H_{e q}\left(e^{j \omega}\right)\right|^{2}=1$ (perfect equalization). The pdf of $L_{o}^{E}$ is assumed to also have a Gaussian distribution with mean and variance

$$
\begin{aligned}
\mu_{L_{o}^{E}} & =E\left\{L_{o}^{E}\right\}=\frac{2\left(h_{0}^{e q} \cdot x_{n}+\epsilon\right)}{\sigma_{n_{o}}^{2}} \\
\sigma_{L_{o}^{E}} & =E\left\{\left(L_{o}^{E}\right)^{2}\right\}-\mu_{L_{o}^{E}}^{2}=\frac{4}{\sigma_{n_{o}}^{2}} .
\end{aligned}
$$

Hence, $L_{o}^{E}$ values have the following distribution conditioned on $\mathbf{x}$,

$$
f_{L_{o}}(l \mid \mathbf{x})=\mathcal{N}\left(\frac{2\left(h_{0}^{e q} \cdot x_{n}+\epsilon\right)}{\sigma_{n_{o}}^{2}}, \frac{4}{\sigma_{n_{o}}^{2}}\right),
$$

where it is noted that $\epsilon$ is a function of neighboring symbols of $x_{n}$ and is independent of $x_{n}$. Thus, (12) can be written

$f_{L_{o}^{E}}\left(l \mid x_{n}, \mathbf{x}_{0}\right)=f_{L_{o}^{E}}\left(l \mid x_{n}, \epsilon\right)=\mathcal{N}\left(\frac{2\left(h_{0}^{e q} \cdot x_{n}+\epsilon\right)}{\sigma_{n_{o}}^{2}}, \frac{4}{\sigma_{n_{o}}^{2}}\right)$,

where $\epsilon=\left(h^{e q}\right)^{T} \mathbf{x}_{0}$. We desire the mutual information between $x_{n}$ and the LLR $L_{o}^{E}$,

$$
I(X ; L) \triangleq \sum_{k} \int f\left(x_{n}^{k}, l\right) \log \frac{f\left(x_{n}^{k}, l\right)}{p\left(x_{n}^{k}\right) f(l)} d l,
$$

in terms of $f_{L_{o}}\left(l \mid x_{n}, \epsilon\right)$. Here, $x_{n}^{k} \triangleq \pm 1$ and $k=0,1$ for BPSK. To this end, we have,

$$
\begin{aligned}
f\left(x_{n}^{k}, l\right) & =\sum_{j=1}^{M} f\left(x_{n}^{k}, l \mid \epsilon_{j}\right) p\left(\epsilon_{j}\right)=\frac{1}{M} \sum_{j=1}^{M} f\left(x_{n}^{k}, l \mid \epsilon_{j}\right) \\
& =\frac{1}{M} \sum_{j=1}^{M} \frac{f\left(x_{n}^{k}, l, \epsilon_{j}\right)}{p\left(\epsilon_{j}\right)}=\frac{1}{M} \sum_{j=1}^{M} \frac{f\left(x_{n}^{k}, l, \epsilon_{j}\right)}{p\left(\epsilon_{j}\right) p\left(x_{n}^{k}\right)} p\left(x_{n}^{k}\right),
\end{aligned}
$$

where $M=2^{M_{1}+M_{2}}$. Since $x_{n}^{k}$ and $\epsilon_{j}$ are independent and $p\left(x_{n}^{0}\right)=p\left(x_{n}^{1}\right)=\frac{1}{2}$, we can express $f\left(x_{n}^{k}, l\right)$ in terms of $f\left(l \mid x_{n}^{k}, \epsilon_{j}\right)$

$$
f\left(x_{n}^{k}, l\right)=\frac{1}{M} \sum_{j=1}^{M} \frac{f\left(x_{n}^{k}, l, \epsilon_{j}\right)}{p\left(\epsilon_{j}, x_{n}^{k}\right)} p\left(x_{n}^{k}\right)=\frac{1}{2 M} \sum_{j=1}^{M} f\left(l \mid x_{n}^{k}, \epsilon_{j}\right) .
$$

Using (16) in (14), we obtain,

$$
\begin{gathered}
I(X ; L)=\sum_{k=0}^{1} \int\left\{\frac{1}{2 M} \sum_{j=1}^{M} f\left(l \mid x_{n}^{k}, \epsilon_{j}\right)\right\} \\
\log \frac{\frac{1}{2 M} \sum_{j=1}^{M} f\left(l \mid x_{n}^{k}, \epsilon_{j}\right)}{p\left(x_{n}^{k}\right) f(l)} d l .
\end{gathered}
$$




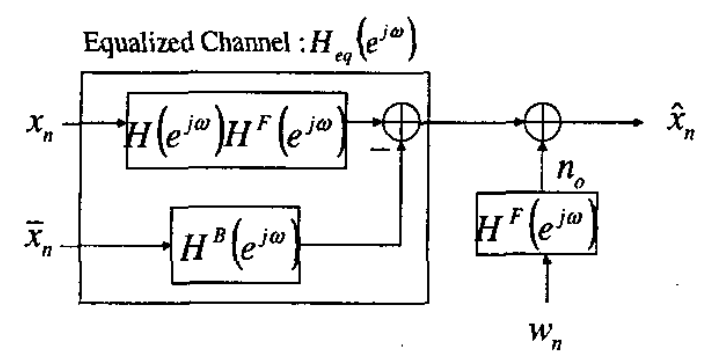

Fig. 7. Equalized channel model including feedback taps, where $H^{B}\left(e^{j \omega}\right)$ is the frequency response of the feedback filter impulse response.

The term, $f(l)$, in (17) can be written

$f(l)=\sum_{i=0}^{1} \sum_{j=1}^{M} f\left(l \mid x_{n}^{i}, \epsilon_{j}\right) p\left(x_{n}^{i}, \epsilon_{j}\right)=\frac{1}{2 M} \sum_{i=0}^{1} \sum_{j=1}^{M} f\left(l \mid x_{n}^{i}, \epsilon_{j}\right)$,

and hence, (17) becomes

$$
\begin{aligned}
I(X ; L) & =\sum_{k=0}^{1} \int\left\{\frac{1}{2 M} \sum_{j=1}^{M} f\left(l \mid x_{n}^{k}, \epsilon_{j}\right)\right\} \\
& \log \frac{2 \sum_{j=1}^{M} f\left(l \mid x_{n}^{k}, \epsilon_{j}\right)}{\sum_{j=1}^{M}\left\{f\left(l \mid x_{n}^{0}, \epsilon_{j}\right)+f\left(l \mid x_{n}^{1}, \epsilon_{j}\right)\right\}} d l,
\end{aligned}
$$

which will be used to compute the mutual information at the two end points of linear SISO equalization EXIT charts.

In order to estimate the EXIT charts of SISO equalizers, the mutual information $I_{o}^{E}$ at the two end points, $I_{i}^{E}=0$ and $I_{i}^{E}=1$, respectively, need to be computed. However, for evaluating the starting and ending points of the linear SISO equalizer EXIT chart, the channel model shown in Fig. 7 should be considered because the feedback filter subtracts ISI effects based on the soft symbol $\bar{x}_{n}$. Under the Gaussian approximation [8], $I_{i}=0$ is equivalent to $L_{i}^{E}=0$ thereby $\bar{x}_{n}=$ 0 . Thus, the feedback path is assumed to make little difference and, using (19), the mutual information of the no a priori information $\left(I_{i}^{E}=0\right)$ case can be computed. In the case of perfect a priori information, $\bar{x}_{n}$ is equal to $x_{n}$. Therefore; by replacing $H_{e q}\left(e^{j \omega}\right)$ of Fig. 5 by $H\left(e^{j \omega}\right) H^{F}\left(e^{j \omega}\right)-H^{B}\left(e^{j \omega}\right)$ of Fig. 7, the mutual information can also be computed using (19). Note that the ISI effects, $\epsilon_{j}$, are different at these two points because the overall channel responses after equalization are different.

\section{B. Analysis via EXIT charts}

The estimated equalizer EXIT chart via computing mutual information at the two end points is projected over the decoder EXIT chart, which is determined given the encoder polynomial [8]. Hence, given channel knowledge, linear equalizer coefficients, and the SISO decoder EXIT chart, the performance of a linear turbo equalizer can be investigated without running the usual extensive simulations. By following the approach in section II-B, we can conclude that 3 iterations are required to converge as shown in Fig. 8 , where the simulation and

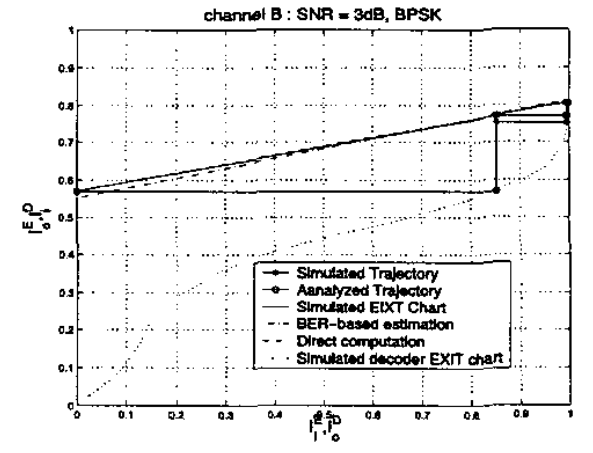

Fig. 8. An example of EXIT chart analysis, where the equalizer EXIT chart is measured via the proposed method and $64 \mathrm{~K}$ interleaver is employed.

analysis results are close to each other but do not match perfectly. The assumption that $L_{i}$ follows the $\mathcal{N}\left(\frac{\sigma_{L_{i}}^{2}}{2}, \sigma_{L_{i}}^{2}\right)$ distribution is simply a model and may be a source of mismatch. Further, since EXIT chart analysis is asymptotic (in that the independence assumptions on LLRs hold for an infinite-length ideal interleaver [5], [8]) and approximate, we expect some mismatch for a finite length interleaver. However, EXIT charts are still useful in predicting the required number of iterations for convergence and the effectiveness of turbo equalization over standard non-iterative equalization.

\section{EXPERIMENTAL RESULTS AND DISCUSSION}

In this section, our proposed analysis results are illustrated with computer simulations.

\section{A. Simulation setup}

We employ a recursive systematic convolutional (RSC) encoder at the transmitter with a generator polynomial $(23,35)_{8}$. The coded bit stream is first passed through a random interleaver followed by a BPSK modulation. For purposes of comparison, we considered two static channel models (channel $\mathbf{A}$ and $\mathbf{B}$ ),

$$
\begin{aligned}
\mathbf{H}_{A}(z)= & 0.04 z^{5}-0.05 z^{4}+0.07 z^{3}-0.21 z^{2}-0.5 z \\
& +0.72+0.36 z^{-1}+0.21 z^{-3}+0.03 z^{-4} \\
& +0.07 z^{-5} \\
\mathbf{H}_{B}(z)= & 0.407 z+0.815+0.407 z^{-1}
\end{aligned}
$$

where $\mathbf{H}_{A}(z)$ is a "good" channel and $\mathbf{H}_{B}(z)$ has more severe ISI [13] (strong spectral null near $\omega=\pi$ ). We use random interleavers and a minimum mean square error criterion is used to determine linear equalizer coefficients [5]. The number of taps used in the feedforward path is 15 and 7 , and the number of taps in the feedback path is 14 and 6 , respectively, for channels A and B. A sliding window Log-MAP SISO decoder [12] is employed and 10 iterations are carried out.

\section{B. Summary of results}

Computer simulations are carried out to validate our analysis and the results are summarized in Table I, where the mutual 
TABLE I

COMPARISON BETWEEN SIMLLATION AND ANALYSIS.

\begin{tabular}{|c||c|c||c|c|}
\hline Channel A & \multicolumn{2}{c|}{ Simulation $\left(I_{e}\right)$} & \multicolumn{2}{c|}{ Analysis $\left(I_{e}\right)$} \\
\hline$E_{B} / N_{0}$ & $1 \mathrm{st}$ & $10 \mathrm{th}$ & $I_{a}^{E}=0$ & $I_{a}^{E}=1$ \\
\hline $1 \mathrm{~dB}$ & 0.718 & 0.759 & 0.721 & 0.763 \\
\hline $2 \mathrm{~dB}$ & 0.782 & $0 . \overline{8} 22$ & 0.785 & 0.825 \\
\hline $3 \mathrm{~dB}$ & 0.838 & 0.874 & 0.843 & 0.879 \\
\hline $4 \mathrm{~dB}$ & 0.886 & 0.916 & 0.891 & 0.921 \\
\hline Channel B & Simulation $\left(I_{e}\right)$ & \multicolumn{1}{|c|}{ Analysis $\left(I_{e}\right)$} \\
\hline$E_{B} / N_{o}$ & $1 \mathrm{st}$ & $10 \mathrm{th}$ & $I_{a}^{E}=0$ & $I_{a}^{E}=1$ \\
\hline $1 \mathrm{~dB}$ & 0.490 & 0.693 & 0.491 & 0.699 \\
\hline $2 \mathrm{~dB}$ & 0.531 & 0.754 & 0.531 & 0.756 \\
\hline $3 \mathrm{~dB}$ & 0.57 & 0.806 & 0.571 & 0.808 \\
\hline $4 \mathrm{~dB}$ & 0.607 & 0.851 & 0.608 & 0.854 \\
\hline
\end{tabular}

TABLE II

THE NLMBER OF ITERATIONS FOR CONVERGENCE FOR CHANNEL A.

\begin{tabular}{|c|c|c|c|c|c|}
\hline \multicolumn{2}{|c|}{ Channel A: 4K interleaver } & \multicolumn{3}{|c|}{ Channel B: $64 \mathrm{~K}$ interleaver } \\
\hline$E_{\mathrm{b}} / N_{o}$ & Simulation & Analysis & $E_{b} / N_{o}$ & Simulation & Analysis \\
\hline $1 \mathrm{~dB}$ & 2 & 2 & $1 \mathrm{~dB}$ & 5 & 4 \\
\hline $2 \mathrm{~dB}$ & 2 & 2 & $2 \mathrm{~dB}$ & 4 & 4 \\
\hline $3 \mathrm{~d} B$ & 2 & 2 & $3 \mathrm{~dB}$ & 3 & 3 \\
\hline $4 \mathrm{~d} \overline{\mathrm{B}}$ & 2 & 2 & $4 \mathrm{~dB}$ & 3 & 3 \\
\hline
\end{tabular}

information is measured both via computer simulations and the proposed method. Our analysis matches well with simulation results. By using the estimated SISO equalizer EXIT charts, the convergence behavior is analyzed and the required number of jterations for each channel is summarized and compared with simulation results in Table II. Note that our analysis, in which an infinite length interleaver is assumed, provides a good estimate of the required number of iterations.

Further, the slope of the estimated equalizer EXIT chart provides a measure of required complexity (the number of iterations needed for convergence). If the slope is steep $\left(\mathbf{H}_{B}(z)\right)$, more iterations are required for convergence and bit error rate (BER) performance improves with iteration as shown in Table III. This means that the output a priori information, $L_{e}^{E}(\cdot)$, becomes more reliable as the input a priori information, $L_{a}^{E}(\cdot)$ becomes more reliable. However, when the slope is less steep $\left(\mathbf{H}_{A}(z)\right)$, more reliable feedback information from the SISO decoder makes little difference in soft outputs. Hence, after a few iterations, BER improvement ceases as shown in Table III. Thus, turbo equalization is more useful in channel $\mathbf{B}$ than in channel $\mathbf{A}$.

\section{CONCLUDING REMARKS}

We propose a linear turbo equalizer convergence analysis method, where the SISO equalizer EXIT chart is estimated, given channel knowledge and a time-invariant set of linear equalizer coefficients. Therefore, without extensive simulations, the required number of jterations for convergence and a measure of equalization complexity are predicted via EXIT charts. Computer simulations support our resuilts.

\section{ACKNOWLEDGMENT}

This material is based upon work supported by the National Science Foundation under Grant CCR 99-79381, ITR 00-
TABLE III

BER MEASLREMENT VIA COMPUTER SIMULATIONS.

\begin{tabular}{|c||c|c|c|c|}
\hline \multicolumn{1}{|c||}{} & \multicolumn{4}{c|}{ Channel A: $4 \mathrm{~K}$ interleaver } \\
\hline$E_{B} / N_{o}$ & $1 \mathrm{st}$ & $2 \mathrm{nd}$ & 3rd & $10 \mathrm{th}$ \\
\hline $1 \mathrm{~dB}$ & 0.0795 & 0.0668 & 0.0668 & 0.0668 \\
\hline $2 \mathrm{~dB}$ & 0.0599 & 0.0481 & 0.0481 & 0.0481 \\
\hline $3 \mathrm{~dB}$ & 0.0433 & 0.0329 & 0.0329 & 0.0329 \\
\hline $4 \mathrm{~dB}$ & 0.0296 & 0.0212 & 0.0212 & 0.0212 \\
\hline \multicolumn{4}{|||}{ Channel B: 64K interleaver } \\
\hline$E_{B} / N_{o}$ & $1 \mathrm{st}$ & $2 \mathrm{nd}$ & $3 \mathrm{rd}$ & $10 \mathrm{th}$ \\
\hline $1 \mathrm{~dB}$ & 0.1612 & 0.1243 & 0.0966 & 0.0871 \\
\hline $2 \mathrm{~dB}$ & 0.1467 & 0.0939 & 0.0709 & 0.0683 \\
\hline $3 \mathrm{~dB}$ & 0.1330 & 0.0678 & $0.053 \mathrm{~J}$ & 0.0526 \\
\hline $4 \mathrm{~dB}$ & 0.1201 & 0.0476 & 0.0395 & 0.0395 \\
\hline
\end{tabular}

85929, and Grant CCR-0092598.

\section{REFERENCES}

[1] C. Berrou, A. Glavieux. and P. Thitimajshima. "Near shannon limit errorcorrecting coding and decoding: Turbo codes," in Proc. of IEEE Int. Conf. on Comm., Geneva. May 1993, pp. 1064-1070.

[2] J. Hagenauer, "Tutorial introduction and state of the art," in Proc Intemarional symposium on Turbo Codes and Related Topics, (Bretagne, France), 1997, pp. 1-11.

[3] P. H. Siegel, D. Divsalar, E. Eleftheriou, J. Hagenauer, and D. Rowitch, "Guest Editorial The Turbo Principle: From theory to practice," IEEE Joumal on Selected Areas in Comm., vol. 19. pp. 793-797, May 2001.

14] A. Glavieux, C. Laot, and J. Labat, "Turbo equalization over a frequency selective channel," in Proc. of Im. Symp. on Turbo Codes \& Related sopics, September 1997, pp. 96-102.

[5] M. Tüchler, R. Kotter, and A. Singer, "Turbo-equalization: principles and new results," IEEE Trans. on Comm., vol. 50, no. 5, pp. 754-767, May 2002.

[6] M. Tüchler, A. Singer, and R. Koetter, "Minimum Mean Squared Error Equalization Using A-Priori Information," IEEE Trans. on Signal Processing, vol. 50, pp. 673-683, Mar. 2002.

[7] M. Tüchler, S. Ten Brink, and J. Hagenauer, "Measures for tracing convergence of iterative decoding algorithms," in Proc. 4th Intemational ITG Conference on Source and Channel coding, Berlin, Germany, Jan. 2002. pp. 53-60.

[8] S. Ten Brink, "Convergence behavior of iteratively decoded parallel concatenated codes," IEEE Trans. on Comm., vol. 49, pp. 1727-1737, October 1999.

[9] A. Roumy, A. J. Grant, I. Fijalkow, P. D. Alexander, and D. Pirez, "TurboEqualization : Convergence Analysis," in Proc. of ICASSP, no, 4, 2001, pp. 2645-2648.

[10] D. Divsalar, S. Dolinar, and F. Pollarar, "Iterative turbo decoder analysis based on density evolution," IEEE Journal on Selected Areas in Communications, vol. 19, pp. 891-907, May 2001.

[11] J. Nelson, A. Singer, and R. Koetter, "Linear turbo equalization for parallel ISI channels," IEEE Transaction on Communicasion, vol. $51, \mathrm{pp}$. $860-864$, June 2003.

[12] A. J. Viterbi, "An Intuitive Justification and a Simplified Implementation of the MAP Decoder for Convolutional Codes," IEEE Joumal on Selected Areas in Communications, vol. 16, no. 2, pp. 260-264, Feb. 1998.

[13] J. G. Proakis, Digital Communications, 3rd ed. McGRAW-HILL, 1995. 